\title{
A systematic approach to the recurrent laryngeal nerve dissection at the cricothyroid junction
}

\author{
Oleksandr Butskiy ${ }^{1,2^{*}}$ D, Brent A. Chang ${ }^{1}$, Kimberly Luu', Robert M. McKenzie ${ }^{1}$ and Donald W. Anderson ${ }^{1}$
}

\begin{abstract}
Background: To describe and evaluate a four step systematic approach to dissecting the recurrent laryngeal nerve (RLN) starting at the cricothyroid junction during thyroid surgery (subsequently referred to as the retrograde medial approach).

Methods: All thyroidectomies completed by the senior author between August 2014 and January 2016 were retrospectively reviewed. Patients were excluded if concurrent lateral or central neck dissection was performed. A follow up period of 1 year was included.

Results: Surgical photographs and illustrations demonstrate the four steps in the retrograde medial approach to dissection of the RLN in thyroid surgery.

Three hundred forty-two consecutive thyroid surgeries were performed in 17 months, including 213 hemithyroidectomies, 91 total thyroidectomies, and 38 completion thyroidectomies. The rate of temporary and permanent hypocalcemia was 13\% (95\% confidence interval [CI]: 8-20\%) and 3\% (95\% Cl: 1-8\%) respectively. The rate of temporary and permanent vocal cord palsy was $9 \%(95 \% \mathrm{Cl}: 6-12 \%)$ and $0.3 \%$ (95\% Cl: $0.01-2 \%)$ respectively. The median surgical times for hemithyroidectomy, total thyroidectomy, and completion thyroidectomy were 39 min (Interquartile range [IQR]: 33-47 min), $48 \mathrm{~min}$ (IQR: 40-60 min), and $40 \mathrm{~min}$ (IQR: 35-51 min) respectively. 1\% of cases required conversion to an alternative surgical approach.

Conclusion: In a tertiary endocrine head and neck practice, the routine use of the retrograde medial approach to RLN dissection is safe and results in a short operative time, and a low conversion rate to other RLN dissection approaches.
\end{abstract}

Keywords: Surgical technique, Thyroidectomy, Recurrent laryngeal nerve, Retrograde dissection, Surgical anatomy

\section{Background}

Thyroid surgeries are the most frequent operations performed by head and neck surgeons in the United States [1]. 2015 American Thyroid Association Guidelines recommend "Visual identification of the recurrent laryngeal [RLN] during dissection in all cases" on moderate-quality evidence [2]. Three approaches to RLN identification have been described: lateral approach, inferior approach, and the superior approach [3].

The lateral approach is routinely used by most surgeons for uncomplicated thyroid surgery and was

\footnotetext{
* Correspondence: butskiy.alex@gmail.com

${ }^{1}$ Division of Otolaryngology - Head \& Neck Surgery, University of British Columbia, Vancouver, BC, Canada

${ }^{2}$ Gordon \& Leslie Diamond Health Care Centre, 4th. Fl. 4299B-2775 Laurel Street, Vancouver, BC V5Z 1M9, Canada
}

advocated since the time of Theodor Kocher (Fig. 1) [4]. In this approach the thyroid lobe is retracted medially, middle thyroid vein is divided, and the RLN is identified at the mid-polar level.

For revision cases and for goiter surgery, the inferior approach is often used. In this approach the RLN is found in the soft areolar tissue in the tracheoesophageal groove proximal to the inferior thyroid artery crossing point. One advantage of this technique is that the RLN is found proximally prior to extra-laryngeal branching and away from thyroid bed scarring that might have been caused by prior surgery [3].

The superior Superior approach is the least used approach. In this approach, the RLN is identified as it enters under the inferior constrictor muscle proximal to 


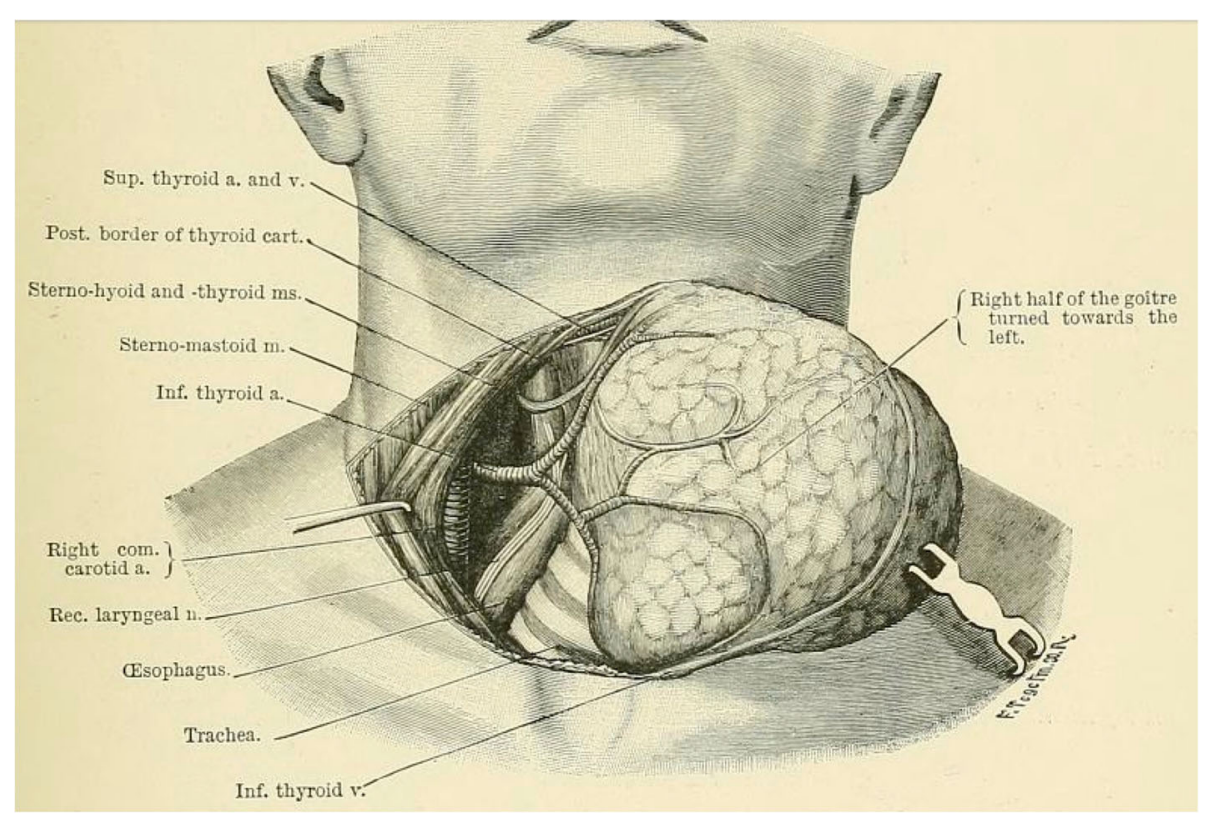

Fig. 1 Illustration to Theodor Kocher's 1895 surgical textbook demonstrating the dissection in the tracheo-esophageal groove after the thyroid is swept medially [4]

the cricothyroid junction [3]. This approach is advantageous as the RLN position relative to the cricothyroid junction is consistent regardless of thyroid pathology and congenital variations [5-8].

The surgical technique for the superior approach has not been described in sufficient detail and its outcomes have not been documented well in literature. Only three studies report on the routine use of the superior approach: one study used a single sentence to describe the surgical technique [9], and the authors of the other two studies followed a technique that appears to be a modification of the lateral approach $[6,10]$. In this technique the thyroid is retracted medially, and the RLN is found by searching between Zukerkandl's tubercle and the cricopharyngeus muscle [6]. Thus, the RLN is not found right at the cricothyroid junction. While a method of identifying the RLN at the cricothyroid junction is likely known to experienced thyroid surgeons, this method has not been described in detail nor evaluated previously in literature.

The main objective of this study was to describe a four step standardized surgical technique to identifying the RLN at the cricothyroid junction (subsequently referred to as the retrograde medial approach). The primary outcome of interest was the rate of surgical complications (temporary and permanent hypocalcemia and vocal cord palsy). The secondary outcomes were surgical time and conversion rate to other techniques.

\section{Methods}

The University of British Columbia (UBC) Research Ethics Board granted approval (H15-01667) for the study.
STROBE Statement for cohort studies was followed in reporting the study [11]. Study was designed as a retrospective review of a cohort of consecutive thyroidectomy patients. All eligible patients were referred to a tertiary head and neck surgical practice affiliated with the University of British Columbia (UBC) for consideration of a thyroidectomy.

All thyroidectomies were performed at teaching hospitals where residents assumed increasing responsibility to complete the procedure as their experience developed. The operations were performed between August 2014 and January 2016.

\section{Participants}

All patients who underwent a hemi-, total, or completion thyroidectomy between August 2014 and January 2016 were selected. To obtain an estimate of the surgical time attributed solely to thyroid dissection, patients were excluded if concurrent lateral or central neck dissection was performed. Pre-operatively all patient underwent visual examination of the larynx, but PTH, Calcium and Vitamin D levels were not routinely measured.

\section{Post-operative care}

After hemithyroidectomy, all patients were observed for 4 hours and were discharged home barring complications. Completion thyroidectomy and total thyroidectomy patients were admitted overnight and their ionized calcium level was checked in the morning. Patients were discharged if the ionized calcium level was above 
$1.00 \mathrm{mmol} / \mathrm{L}$. Routine calcium/vitamin D supplementation and surgical drains were not used.

\section{Follow-up}

Post-operative follow-up consisted of an office visit 2 weeks after the operation or earlier on patient request. Post-operative larynx visualization was performed only if patients described voice or swallowing abnormalities on specific questioning. If any abnormality was detected on the first post-operative visit, such as hypocalcemia or vocal cord palsy, the patients were followed monthly for up to a year or until the abnormality resolved.

\section{Data collection, variables and data sources}

Four co-investigators (OB, BAC, RMM, and KL) collected data retrospectively from August 2016 to May 2017. Patient demographic and surgical indications data was obtained from clinic notes. Procedures performed, surgical times, and admission duration data were obtained from hospital records. Surgical time was defined as the time from first incision to wound closure completion as documented by the nursing staff.Operative dictations were used to establish if conversion to an alternative RLN dissection technique occurred. Pathological diagnoses and thyroid weights were determined reviewing the final pathology reports.

Data on the following complications were extracted from hospital and clinic records: temporary and permanent hypocalcemia and vocal cord palsy, hematoma, seroma, wound infection, and subcutaneous emphysema. Superior laryngeal neve palsy was not looked for during the follow up visit unless deemed necessary. Temporary hypocalcemia was defined as any hypocalcemia requiring calcium or vitamin D supplementation within 6 months of surgery. Permanent hypocalcemia was defined as any hypocalcemia requiring supplementation for greater than
6 months after surgery. Temporary vocal cord palsy was defined as the loss of true vocal cord adduction lasting less than 6 months of surgery, after which the palsy was deemed permanent. Hematoma was defined as any bleeding requiring reoperation or drain placement. In addition, the provincial health care database was screened for post-operative complications related hospital visits that might not have been captured in clinic charts. Patients with missing data were included in the study.

\section{Surgical technique}

Skin incision, elevation of subplatysmal flaps, separation of strap muscles, and release of the sternothyroid muscles to expose the thyroid gland were performed in the usual manner. Unless invaded by the tumor, sternothyroid muscles were not cut. All vessel ligation was performed with Adson insulated bipolar forceps (Kirwan Surgical Products, Marshfield, MA) without routine use of suture ties or surgical clips. RLN monitor was not used. The next four steps are critical to the described technique.

\section{Isolation and division of the isthmus:}

The thyroid isthmus is isolated and divided over the trachea using bipolar cautery.

2. Subtotal division of Berry's suspensory ligaments and exposure of the cricothyroid region (Fig. 2):

The divided edge of the isthmus is grasped with Babcock clamps and retracted laterally (Fig. 2a). Inferior to the first tracheal ring, Berry's suspensory ligaments are divided to separate the thyroid away from the trachea (Fig. 2b). Retraction of the trachea toward the contralateral side

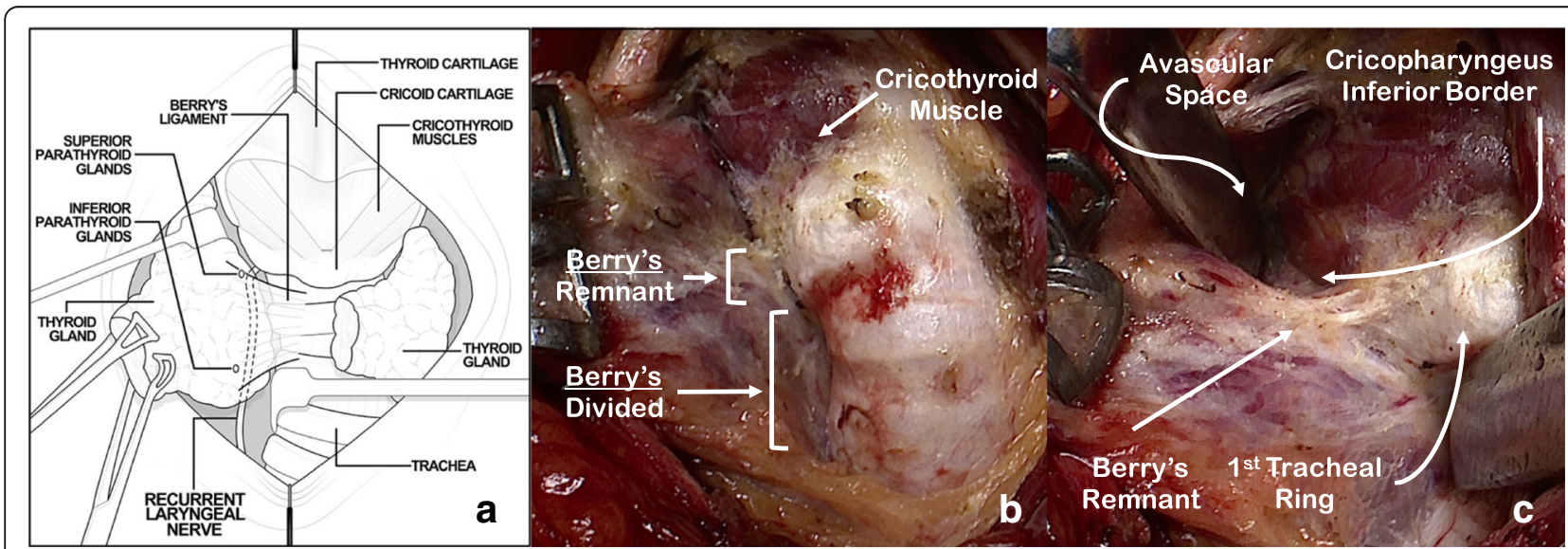

Fig. 2 Subtotal division of Berry's suspensory ligaments and exposure of the cricothyroid region. a Schematic demonstrating anatomy and instrument orientation; b Subtotal division of Berry's ligaments; c Placing Berry's ligament remnant on the stretch with avascular space open 
facilitates performance of this step. Care is taken to dissect directly on the trachea, as the RLN will be protected by a layer of fascia and ligamentous tissue. Lateral to the first tracheal ring the full thickness of Berry's suspensory ligament is left intact (Fig. 2c).

The avascular space between the superior pole and the cricothyroid muscle is developed avoiding damage to the superior laryngeal nerve and the cricothyroid muscle (Fig. 2c). Differential traction between the superior pole and the trachea (demonstrated by the Langenbeck retractors in Fig. 2a \& c) is critical for exposure and identification of the anatomical structures. Bowstring tension promotes isolation and identification of the Berry's ligament remnant. Subsequent to injury-free identification of the cricothyroid muscle, care is then taken to expose the inferior border of the cricopharyngeus muscle, a critical landmark for RLN identification (Fig. 2c).

3. Identification and dissection of the recurrent laryngeal nerve (Fig. 3):

At this point, the remnant of the Berry's ligament tethers the thyroid to the superior portion of the first tracheal ring and protects the RLN from the stretch of the retractors (Fig. 3a). Deep to the remnant are the RLN and the inferior thyroid artery terminal branches. The RLN runs in a plane posterior to Berry's ligaments and then turns medially to enter the larynx at the superior aspect of the ligamentous tissue, forming an anatomical 'genu' [8]. Careful dissection through the superior portion of this ligamentous tissue using a fine mosquito forceps and bipolar cautery allows identification of the RLN insertion under the inferior constrictor (Fig. 2b). Inferior border of the cricopharyngeus muscle is visualized at all times and serves as a landmark for the depth at which the RLN is found. Dissection is facilitated by judicious and careful control of the inferior thyroid artery terminal branches to avoid bleeding that can make RLN visualization difficult. We use bipolar cautery to control the terminal branches, with tips cooled with a wet gauze between applications. As the remnant of the Berry's ligament is transected, the retraction is reduced to avoid stretch injury to the RLN. Once the RLN branches are identified, they are traced inferiorly by dividing the remainder of the Berry's suspensory ligament and releasing the RLN from the thyroid gland. The maximum extent of RLN dissection is 1 to $2 \mathrm{~cm}$ (Fig. 3c).

4. Capsular dissection with preservation of parathyroid tissue and ligation of superior pole vessels (Fig. 4):

The thyroid gland is then swept medially, delivered through the wound, and retracted in an anterosuperior direction (Fig 4a). With the RLN in direct view, a capsular dissection is undertaken from inferior to superior direction. Elevation of the gland assists in exposure and isolation of the blood vessels feeding the parathyroid glands, helping their preservation under direct visualization (Fig. 4a).

The thyroid lobe remains pedicled on the superior pole vessels (Fig. 4b). Gentle retraction of the lobe inferiorly allows for their ligation and division, releasing the thyroid from the wound (Fig. 4c). Hemostasis is achieved and multi-layered closure is performed in the usual fashion.

\section{Results}

Three hundred sixty-seven operations were eligible for inclusion. Twenty-five operations were excluded as either a central or a lateral neck dissection was performed concurrently. Out of 342 surgeries included in the analysis, 213 (62\%) were hemi-, 91 (27\%) were total, and 38

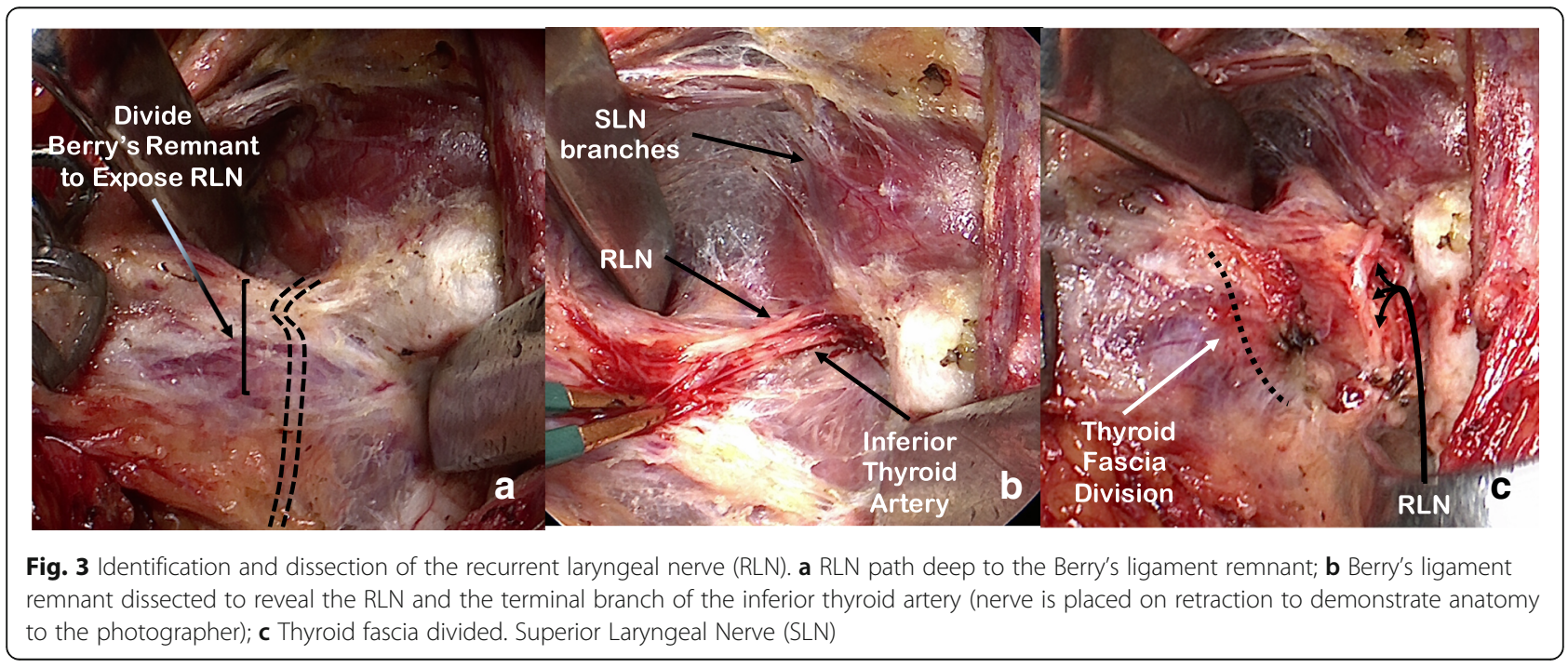




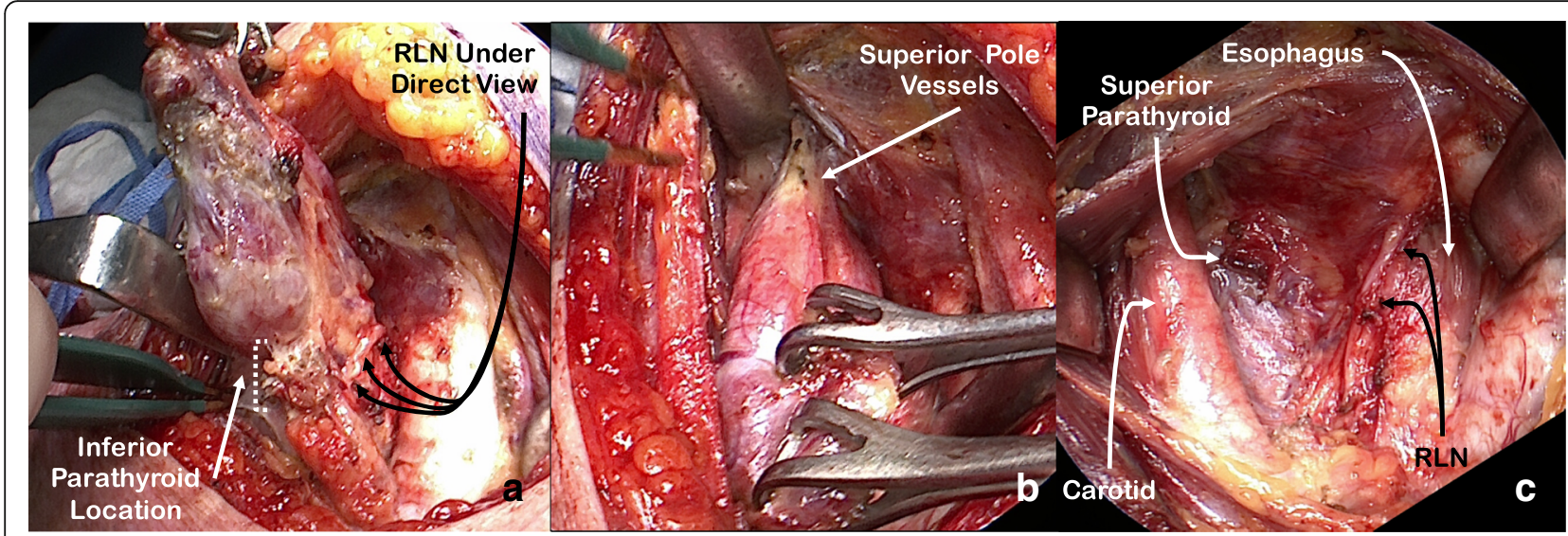

Fig. 4 Capsular dissection with preservation of parathyroid tissue and ligation of superior pole vessels. a Identification of parathyroid glands while keeping the recurrent laryngeal nerve (RLN) under direct view; b Isolation and ligation of superior pole vessels; c Surgical bed after resection

(11\%) were completion thyroidectomies. The mean age of the patients was 50 years (range: $13-89$ years), and $84 \%$ of patients were female.

The most common indications for a total thyroidectomy were papillary thyroid carcinoma (40\%) and thyromegaly (27\%) (Table 1). The most common indications for a hemithyroidectomy were nodules of intermediate suspicion for malignancy (54\%) and thyromegaly (38\%) (Table 1).

The final pathology results showed a variety of benign and malignant conditions (Table 2). The median surgical times for a total, hemi-, and completion thyroidectomy were $48 \mathrm{~min}$ (Interquartile range [IQR]: 40-60 $\mathrm{min}$ ),
39 min (IQR: $33-47 \mathrm{~min}$ ) and $40 \mathrm{~min}$ (IQR: $35-51 \mathrm{~min}$ ) respectively (Table 3 ).

Complications experienced by the patients are summarized in Table 4. Out of 129 patients who underwent total or completion thyroidectomy, 17 (13\%; 95\% confidence interval $[\mathrm{CI}]: 8-20 \%)$ experienced transient and 4 (3\%; 95\% CI:1-8\%) experienced permanent hypocalcemia. Out of 342 patients, 30 (9\%; 95\% CI: 6-12\%) experienced temporary (i.e. $7 \%$ of nerves at risk), and 1 experienced permanent vocal cord palsy $(0.3 \%$; $95 \% \mathrm{CI}$ : $0.01-2 \%)$. There were no instances of bilateral vocal cord palsy. It was unclear what caused the majority of temporary vocal cord palsies. In a minority of cases of

Table 1 Indications for surgery

\begin{tabular}{|c|c|c|c|c|}
\hline & $\begin{array}{l}\text { Total thyroidectomy } \\
n=91\end{array}$ & $\begin{array}{l}\text { Hemi-thyroidectomy } \\
n=213\end{array}$ & $\begin{array}{l}\text { Completion thyroidectomy } \\
n=38\end{array}$ & $\begin{array}{l}\text { Total } \\
n=342\end{array}$ \\
\hline \multicolumn{5}{|l|}{ Nodules requiring diagnosis } \\
\hline Intermediate suspicion for malignancy & - & $114(54 \%)$ & $2(5 \%)$ & $116(34 \%)$ \\
\hline High suspicion for malignancy & $15(16 \%)$ & $2(1 \%)$ & $1(3 \%)$ & $18(5 \%)$ \\
\hline \multicolumn{5}{|l|}{ Thyromegaly } \\
\hline Obstructive/Symptomatic & $20(22 \%)$ & $73(34 \%)$ & $6(16 \%)$ & $99(29 \%)$ \\
\hline Substernal goiter & $5(5 \%)$ & $8(4 \%)$ & - & $14(4 \%)$ \\
\hline \multicolumn{5}{|l|}{ Malignancy } \\
\hline Papillary carcinoma & $36(40 \%)$ & $2(1 \%)$ & $17(45 \%)$ & $55(16 \%)$ \\
\hline Follicular carcinoma & - & - & $8(21 \%)$ & $8(2 \%)$ \\
\hline Poorly differentiated carcinoma & - & - & $1(3 \%)$ & $1(0.3 \%)$ \\
\hline Lymphoma & - & - & $1(3 \%)$ & $1(0.3 \%)$ \\
\hline \multicolumn{5}{|l|}{ Endocrinological diseases } \\
\hline Graves' disease & $13(14 \%)$ & $1(0.5 \%)$ & $1(3 \%)$ & $15(4 \%)$ \\
\hline Hashimoto thyroiditis & $1(1 \%)$ & $2(1 \%)$ & - & $3(1 \%)$ \\
\hline \multicolumn{5}{|l|}{ Other } \\
\hline Symptomatic cyst & - & $9(4 \%)$ & - & $9(3 \%)$ \\
\hline PET incidentaloma & $1(1 \%)$ & $2(1 \%)$ & - & $3(1 \%)$ \\
\hline
\end{tabular}


Table 2 Pathological diagnoses

\begin{tabular}{|c|c|c|c|c|}
\hline & $\begin{array}{l}\text { Total thyroidectomy } \\
(n=91)\end{array}$ & $\begin{array}{l}\text { Hemi-thyroidectomy } \\
(n=213)\end{array}$ & $\begin{array}{l}\text { Completion thyroidectomy } \\
(n=38)\end{array}$ & $\begin{array}{l}\text { Total } \\
(n=342) \\
\end{array}$ \\
\hline \multicolumn{5}{|l|}{ Malignancies } \\
\hline Papillary & $50(54 \%)$ & $22(10 \%)$ & $12(32 \%)$ & $84(25 \%)$ \\
\hline Follicular & $1(1 \%)$ & $11(5 \%)$ & $2(5 \%)$ & $14(4 \%)$ \\
\hline Poorly differentiated & $1(1 \%)$ & $1(0.5 \%)$ & - & $2(1 \%)$ \\
\hline B-Cell lymphoma & - & $1(0.5 \%)$ & - & $1(0.3 \%)$ \\
\hline Renal cell carcinoma & $1(1 \%)$ & - & - & $1(0.3 \%)$ \\
\hline \multicolumn{5}{|l|}{ Goiters } \\
\hline Multinodular goiter & $16(18 \%)$ & 73 (34\%) & $9(24 \%)$ & $98(29 \%)$ \\
\hline Diffuse goiter & $1(1 \%)$ & $5(2 \%)$ & $1(3 \%)$ & $7(2 \%)$ \\
\hline \multicolumn{5}{|l|}{ Endocrine diseases } \\
\hline Graves' disease & $8(9 \%)$ & $1(0.5 \%)$ & - & $9(3 \%)$ \\
\hline Hashimoto's thyroiditis & $7(8 \%)$ & $10(5 \%)$ & $1(3 \%)$ & $18(5 \%)$ \\
\hline \multicolumn{5}{|l|}{ Other benign pathology } \\
\hline Follicular adenoma & $4(4 \%)$ & $65(31 \%)$ & $1(3 \%)$ & $70(20 \%)$ \\
\hline Thyroid cyst & - & $5(2 \%)$ & - & $5(2 \%)$ \\
\hline Not otherwise specified & $2(2 \%)$ & $19(9 \%)$ & 12 (32\%) & $33(10 \%)$ \\
\hline
\end{tabular}

temporary vocal cord palsy, the dictating surgeon commented on the difficulty due to inflammation and bleeding. In the single instance of permanent vocal cord paralysis, a RLN nerve branch was cut.

In 3 patients (1\%) a conversion to an alternative method of RLN dissection was needed, this was due to difficulty in controlling bleeding (2 cases) and dense scarring superior to Berry's ligament (1 case).

\section{Discussion}

In this study we introduce a systematic approach to dissecting the RLN at the cricothyroid junction, referred to here as the retrograde medial approach. The outcomes of this approach were investigated through a retrospective review of a single surgeon's cohort of patients. The rates of transient and permanent vocal cord palsy were 9\% (95\% CI: $6-12 \%$ ) and $0.3 \%$ (95\%CI: $0.01-2 \%$ ) respectively, while the rates of transient and permanent hypocalcemia were
13\% (95\% CI: 8-20\%) and 3\% (95\% CI: 1-8\%) respectively. The retrograde medial approach appears to be fast, with a median surgical time of $41 \mathrm{~min}$.

Three previous studies have described using the superior approach to finding the RLN. While the authors of one study do not describe the specific technique [9], the authors of the other two studies followed the technique first described by Shindo et al $[6,10]$. This technique involves releasing the superior pole, finding and releasing the tubercle of Zuckerkandl, retracting the gland medially, and then searching for the RLN as it courses towards the cricothyroid junction. Shindo et al. acknowledged the variability in the angle that the RLN takes as it approaches the cricothyroid junction and classified it into four categories. The authors also acknowledged that their technique is difficult in cases of nonrecurrent RLN, presence of large tubercle of Zuckerkandl, and extrathyroidal extension of cancer along the distal RLN segment.

Table 3 Surgical times

\begin{tabular}{|c|c|c|c|c|}
\hline & $\begin{array}{l}\text { Total thyroidectomy } \\
\text { Median (IQR) }\end{array}$ & $\begin{array}{l}\text { Hemi-thyroidectomy } \\
\text { Median (IQR) }\end{array}$ & $\begin{array}{l}\text { Completion thyroidectomy } \\
\text { Median (IQR) }\end{array}$ & $\begin{array}{l}\text { Total } \\
\text { Median (IQR) }\end{array}$ \\
\hline All indications & $48 \min (40-60 \mathrm{~min})$ & $39 \min (33-47 \mathrm{~min})$ & $40 \min (25-93 \mathrm{~min})$ & $41 \min (35-51 \mathrm{~min})$ \\
\hline Nodules requiring diagnosis & $42 \min (39-50 \mathrm{~min})$ & $38 \min (32-44 \min )$ & $29 \min (27-56 \min )$ & $39 \min (32-45 \mathrm{~min})$ \\
\hline \multicolumn{5}{|l|}{ Thyromegaly } \\
\hline Obstructive/Symptomatic & $57 \min (47-66 \mathrm{~min})$ & $41 \min (35-50 \mathrm{~min})$ & $41 \min (33-54 \min )$ & $44 \min (37-53 \mathrm{~min})$ \\
\hline Substernal goiter & $84 \min (50-98 \min )$ & $60 \min (52-65 \min )$ & $93 \min$ & $64 \min (51-81 \mathrm{~min})$ \\
\hline Malignancy & $47 \min (40-53 \mathrm{~min})$ & $51 \min (45-55 \min )$ & $39 \min (36-46 \min )$ & $43 \min (38-51 \mathrm{~min})$ \\
\hline Graves' disease & $55 \min (43-63 \mathrm{~min})$ & $60 \min$ & $67 \min$ & $56 \min (43-65 \mathrm{~min})$ \\
\hline
\end{tabular}


Table 4 Course in hospital and complications

\begin{tabular}{|c|c|c|c|c|}
\hline & $\begin{array}{l}\text { Total thyroidectomy } \\
n=91\end{array}$ & $\begin{array}{l}\text { Hemi-thyroidectomy } \\
n=213\end{array}$ & $\begin{array}{l}\text { Completion thyroidectomy } \\
n=38\end{array}$ & $\begin{array}{l}\text { Total } \\
n=342\end{array}$ \\
\hline Nights in hospital - median (Range) & $1(0-30)$ & $0(0-3)$ & $1(1-7)$ & $1(0-30)$ \\
\hline Conversion to alternative approach - n (\%) & $2(2 \%)$ & - & $1(3 \%)$ & $3(1 \%)$ \\
\hline \multicolumn{5}{|l|}{ Complications - n (\%) } \\
\hline Transient hypocalcemia & $16(18 \%)$ & - & $1(3 \%)$ & $17(5 \%)$ \\
\hline Permanent hypocalcemia & $1(1 \%)$ & - & $2(5 \%)$ & $3(1 \%)$ \\
\hline Temporary vocal cord paresis/Paralysis & $13(14 \%)$ & $14(7 \%)$ & $3(8 \%)$ & $30(9 \%)$ \\
\hline Permanent vocal cord paresis/Paralysis & $1(1 \%)$ & - & - & $1(0.3 \%)$ \\
\hline Hematoma & $1(1 \%)$ & $4(2 \%)$ & $1(3 \%)$ & $6(2 \%)$ \\
\hline Seroma & - & $1(0.5 \%)$ & - & \\
\hline Wound infection & $5(6 \%)$ & $3(1 \%)$ & $1(2.6 \%)$ & $9(3 \%)$ \\
\hline Subcutaneous emphysema & $1(1 \%)$ & $2(1 \%)$ & - & $3(1 \%)$ \\
\hline
\end{tabular}

In the presented retrograde medial approach, the RLN nerve is found early prior to the release of the superior pole and exploration of the thyroid's lateral side. Given that the RLN is found superior to the Berry's ligament, the variability in the angle that RLN takes in its approach has no impact on the dissection. Furthermore, the retrograde medial approach is preferential in cases with nonrecurrent RLN, large Zuckerkandl's tubercle, and in the majority of cases with extrathyroidal extension of cancer along the distal RLN segment (with exception of cricothyroid junction involvement). We find the retrograde medial approach especially useful in cases of large goiters. Finally, an additional advantage of the retrograde medial approach is that no thyroid tissues is left unresected at the cricothyroid junction.

Some surgical situations make the use of retrograde medial approach difficult. The method relies on splitting the thyroid isthmus and identifying the inferior border of the cricopharyngeal muscle. If a surgeon is unable to complete these steps, either due to fear of tumor spillage with the isthmus division or extensive scarring, bleeding, or presence of tumor at the cricopharyngeus muscle or the cricothyroid junction, it is advisable that the lateral or inferior approach to the thyroidectomy is used.

With the use of the retrograde medial approach, the rates of permanent vocal cord palsy and hypocalcemia are similar to the rates reported with other superior approaches to finding the RLN; however, the rates of temporary vocal cord palsy and hypocalcemia appear higher. With regard to the permanent complications, in a study of 181 patients, Sykes et al. reported permanent vocal cord palsy and hypocalcemia rates of $0.4 \%$ and $2.2 \%$ respectively [10]. In a study of 67 patients, Veyseller et al. reported the corresponding rates to be 0 [9].
With regard to the transient complications, Sykes et al. reported a $2.2 \%$ rate of temporary vocal cord palsy [10]; whereas Veyseller et al. reported temporary vocal cord palsy and hypocalcemia rates of $0 \%$ and $8.3 \%$ respectively [9].

There are a number of possible reasons to why the rates of temporary complications were higher in the presented study than in the studies discussed above. First, given different sizes and compositions of populations studied, the difference could be secondary to sampling variability. Second, the difference might be due to different definitions of temporary complications. Specifically, compared to the mentioned studies we used a more liberal definition of hypocalcemia - requirement for calcium or vitamin $\mathrm{D}$ supplementation regardless of the reason. Finally, the technique described in this paper could be the reason for higher temporary complication rates. For example, the exclusive use of bipolar cautery could be responsible for transient RLN and parathyroid gland heat damage. Some surgeons might also suggest that traction at the Berry's ligament transferred to the RLN might be responsible for higher transient vocal cord palsy rate. However, there is no tension on the nerve until the suspensory ligament is divided. Recognizing this is a point of importance and teaching. After division of the suspensory ligament the nerve is dissected out from top to bottom while on the slack at the fixation point at entrance to larynx.

Comparing the outcomes of the retrograde medial technique to the outcomes of the two other approaches (lateral and inferior) is difficult given that one approach is rarely used exclusively. Perhaps the best estimate of thyroidectomy complication rates comes from national studies. For example, the 2008 study of Scandinavian Quality Register for Thyroid and Parathyroid Surgery 
reported complication rates based on 3660 thyroidectomies [12]. In this report, the authors presented data on hypocalcemia defined identically to our study - the use of supplemental calcium and/or vitamin $\mathrm{D}$ at the first post-operative visit and 6 months after surgery. Using this definition, the rates of transient and permanent hypocalcemia in the Scandinavian study (17\% and 6\% respectively) appear similar to our study (13\% and $3 \%$ respectively). With regard to vocal cord palsy, the authors of the Scandinavian study report temporary palsy rates lower than in our study (3.9\% versus $9 \%$ respectively), but the permanent palsy rates appear similar to our study $(0.9 \%$ versus $1 \%$ respectively).

With regard to surgical time, the authors of the studies described above do not report on the surgical speed. The speed of the described technique appears similar to the techniques described in recent meta-analyses and reviews of ultrasonic and electrothermal devices in thyroid surgery $[13,14]$. For example, the authors of a recent meta-analysis concluded that a total thyroidectomy is performed faster with the use of Harmonic Focus ${ }^{\circ}$ (Ethicon Inc., Cincinnati $\mathrm{OH}$ ) than with the use of clips and ties [13]. The pooled average time for a total thyroidectomy using the Harmonic Focus ${ }^{\ominus}$ was 66 min and the average time for the conventional technique was $95 \mathrm{~min}$ [13]. In comparison, the average time for a total thyroidectomy in our series was $48 \mathrm{~min}$.

Short operative time with the retrograde medial approach is possibly due to early RLN identification in a consistent location. The remaining dissection can then proceed quickly without concern of RLN injury. This is in contrast to the lateral approach in which complete RLN dissection is one of the last operating steps. We acknowledge that aside from the RLN dissection technique there might be other reasons for shorter operative time. These include the use of bipolar cautery in place of clips and ties and the surgical experience of the author.

The presented study has strengths. First, the described approach, while likely known to experienced thyroid surgeons, has not been previously reported in surgical literature. Second, while the presented technique is not meant to be prescriptive, prior to this study there has been no attempt to standardize identification of RLN at the cricothyroid junction. Finally, this study reports on the largest cohort of patients in literature for whom the superior approach to identifying RLN was used.

This series has limitations. First, a retrospective review might have resulted in missing data for complications. . Second, the study represents a single surgeon's experience, possibly limiting the generalizability of the findings. Finally, our study excluded patients requiring lateral and central neck dissection; therefore, further investigation is required to determine the utility of the retrograde medial approach in such cases.

\section{Conclusion}

A retrograde medial approach to identifying the RLN at the cricothyroid junction is described. This technique is useful in dissecting large goiters and when lateral RLN identification is difficult. In a tertiary endocrine head and neck practice, the routine use of the retrograde medial approach is safe and results in short operative time and a low conversion rate to other RLN dissection approaches.

\section{Acknowledgment \\ We would like to thank Mr. Callum Faris for encouragement to publish the retograde medial approach and his assistance with study design.}

\section{Availability of data and materials}

The datasets used and/or analysed during the current study are available from the corresponding author on reasonable request.

\section{Authors' contributions}

All authors contributed to study design. All authors with exception of DWA participated in data collection. OB analyzed the data and prepared the manuscript. All other authors edited the manuscript for publication. All authors read and approved the final manuscript.

Ethics approval and consent to participate

Institutional ethics approval was granted for the project (H15-01667).

Consent for publication

Not applicable

\section{Competing interests}

The authors declare that they have no competing interests.

\section{Publisher's Note}

Springer Nature remains neutral with regard to jurisdictional claims in published maps and institutional affiliations.

Received: 14 April 2018 Accepted: 3 September 2018 Published online: 17 September 2018

\section{References}

1. Bhattacharyya $\mathrm{N}$. The increasing workload in head and neck surgery: an epidemiologic analysis. Laryngoscope. 2011;121(1):111-5. https://doi.org/10. 1002/lary.21193.

2. Haugen BR, Alexander EK, Bible KC, et al. 2015 American thyroid association management guidelines for adult patients with thyroid nodules and differentiated thyroid cancer: the American thyroid association guidelines task force on thyroid nodules and differentiated thyroid cancer. Thyroid. 2015;26(1):1-133. https://doi.org/10.1089/thy.2015.0020.

3. Richer SL, Randolph GW. Management of the recurrent laryngeal nerve in thyroid surgery. Oper Tech Otolaryngol-Head Neck Surg. 2009;20(1):29-34. https://doi.org/10.1016/j.otot.2009.02.006.

4. Kocher T, Stiles HJ. Text-book of operative surgery. London: Adam and Charles Black; 1895. http://archive.org/details/textbookofoperat00koch

5. Çakir BÖ, Ercan I, Şam B, Turgut S. Reliable surgical landmarks for the identification of the recurrent laryngeal nerve. Otolaryngol Head Neck Surg. 2006;135(2):299-302. https://doi.org/10.1016/j.otohns.2006.03.026.

6. Shindo ML, Wu JC, Park EE. Surgical anatomy of the recurrent laryngeal nerve revisited. Otolaryngol Head Neck Surg. 2005;133(4):514-9. https://doi. org/10.1016/j.otohns.2005.07.010.

7. Ardito G, Revelli L, D'Alatri L, Lerro V, Guidi ML, Ardito F. Revisited anatomy of the recurrent laryngeal nerves. Am J Surg. 2004;187(2):249-53. https://doi. org/10.1016/j.amjsurg.2003.11.001.

8. Sasou S, Nakamura S, Kurihara H. Suspensory ligament of berry: its relationship to recurrent laryngeal nerve and anatomic examination of 24 autopsies. Head Neck. 1998;20(8):695-8. https://doi.org/10.1002/(SICI)10970347(199812)20:8<695::AID-HED6>3.0.CO;2-3. 
9. Veyseller B, Aksoy F, Y Y KA, Özturan O. EFfect of recurrent laryngeal nerve identification technique in thyroidectomy on recurrent laryngeal nerve paralysis and hypoparathyroidism. Arch Otolaryngol Neck Surg. 2011;137(9): 897-900. https://doi.org/10.1001/archoto.2011.134.

10. Sykes RF, Moorthy R, Olaleye O, Black IM. Identification of the recurrent laryngeal nerve at the cricothyroid joint: our experience of 181 thyroid procedures. Clin Otolaryngol. 2014;39(3):174-7. https://doi.org/10.1111/coa. 12254.

11. von Elm E, Altman DG, Egger M, et al. The Strengthening the Reporting of Observational Studies in Epidemiology (STROBE) statement: guidelines for reporting observational studies. Ann Intern Med. 2007;147(8):573. https://doi. org/10.7326/0003-4819-147-8-200710160-00010.

12. Bergenfelz A, Jansson S, Kristoffersson A, et al. Complications to thyroid surgery: results as reported in a database from a multicenter audit comprising 3,660 patients. Langenbeck's Arch Surg. 2008;393(5):667-73. https://doi.org/10.1007/s00423-008-0366-7.

13. Cheng H, Soleas I, Ferko NC, Clymer JW, Amaral JF. A systematic review and meta-analysis of harmonic focus in thyroidectomy compared to conventional techniques. Thyroid Res. 2015;8:15. https://doi.org/10.1186/ s13044-015-0027-1.

14. Butskiy O, Wiseman SM. Electrothermal bipolar vessel sealing system (LigaSure) for hemostasis during thyroid surgery: a comprehensive review. Expert Rev Med Devices. 2013;10(3):389-410. https://doi.org/10.1586/erd.13.6.

Ready to submit your research? Choose BMC and benefit from:

- fast, convenient online submission

- thorough peer review by experienced researchers in your field

- rapid publication on acceptance

- support for research data, including large and complex data types

- gold Open Access which fosters wider collaboration and increased citations

- maximum visibility for your research: over $100 \mathrm{M}$ website views per year

At $\mathrm{BMC}$, research is always in progress.

Learn more biomedcentral.com/submissions 